\title{
Estresse: uma realidade vivenciada pelos colaboradores de enfermagem no centro cirúrgico
}

\author{
Stress: a reality experienced by the nursing team in the operating room \\ Estrés: una realidad vivida por el personal de enfermería en el quirófano
}

Aline Silva dos Santos Dapper ${ }^{1 *}$, Josiane Palavro Barros ${ }^{1}$, Marcia Dornelles Machado Mariot ${ }^{2}$, Dayane de Aguiar Cicolella².

\section{RESUMO}

Objetivo: Avaliar o nível de estresse dos profissionais de enfermagem que atuam no centro cirúrgico de uma cidade do Rio Grande do Sul. Métodos: Trata-se de um estudo quantitativo, transversal e descritivo, realizado com 52 profissionais de enfermagem, através do instrumento Inventário de Sintomas de Estresse para adulto de lipp (ISSL). A pesquisa foi realizada de janeiro a junho do ano de 2020, em um Centro Cirúrgico (CC) e Centro de Materiais Esterilizados (CME) de um hospital de médio porte. Os dados foram analisados através do programa SPSS versão 21.0, testes Qui-quadrado e teste t-student. Também foi utilizada Regressão de Poisson. Resultados: Observou-se que, aproximadamente, a metade dos entrevistados apresentava stress $(44,2 \%)$ e a maioria encontravam-se e em fase de resistência com sintoma fator psicológico (60,9\%). Apresentando as variáveis: ter filhos adolescentes $(p=0,014)$ e realizar atividade acadêmica $(p=0,005)$, apresentou fator de risco elevado ao estresse. Conclusão: $O$ estudo permitiu traçar o perfil dos trabalhadores que estão mais predispostos ao stress, apresentando elevados índices de estresse em fase de resistência. Acredita-se que tal situação possa estar relacionada às práticas dos profissionais que geram muita tensão, demostrando haver a necessidade de estratégias que ofereçam apoio à realização de atividade profissional nesses setores.

Palavras-chave: Profissionais de enfermagem, Estresse ocupacional, Centros cirúrgicos.

\section{ABSTRACT}

Objective: To assess the stress level of nursing professionals who work in the operating room of a city in Rio Grande do Sul. Methods: This is a quantitative, cross-sectional and descriptive study, carried out with 52 nursing professionals, using the Inventory of Symptoms of Stressfor lipp adult (ISSL). The research was carried out from January to June of the year 2020, in a Surgical Center (CC) and Sterile Materials Center (CME) of a medium-sized hospital. The data were analyzed using the SPSS software version 21.0, Chi-square tests and Student's t-test. Poisson regression was also used. Results: It was observed that approximately half of the interviewees had stress (44.2\%) and the majority were in a resistance phase with a psychological factor symptom $(60.9 \%)$. Presenting the variables: having adolescent children $(p=0.014)$ and performing academic activity $(p=0.005)$, presented a high risk factor for stress. Conclusions: The study made it possible to trace the profile of workers who are more predisposed to stress, with high levels of stress in the resistance phase. It is believed that such a situation may be related to the practices of professionals that generate a lot of tension, demonstrating the need for strategies that offer support for carrying out professional activity in these sectors.

Key words: Nurse practitioners, Occupational stress, Surgicenters.

\footnotetext{
1 Cesuca Centro Universitário, Cachoeirinha-RS. *E-mail: ss82.santos@gmail.com
}

2 Universidade Federal do Rio Grande do Sul, Porto Alegre - RS. 


\section{RESUMEN}

Objetivo: Evaluar el nivel de estrés de los profesionales de enfermería que laboran en el quirófano de una ciudad de Rio Grande do Sul. Métodos: Se trata de un estudio cuantitativo, transversal y descriptivo, realizado con 52 profesionales de enfermería, a través del instrumento de Inventario de Síntomas de Estrés para adultos lipp (ISSL). La investigación se realizó de enero a junio del año 2020, en un Centro Quirúrgico (CC) y Centro de Materiales Estériles (CME) de un hospital de tamaño mediano. Los datos se analizaron mediante el programa SPSS versión 21.0, pruebas de chi-cuadrado y t de Student, así como regresión de Poisson. Resultados: Se observó que aproximadamente la mitad de los entrevistados presentaba estrés $(44,2 \%)$ y la mayoría se encontraba en fase de resistencia con un síntoma de factor psicológico $(60,9 \%)$. Presentar las variables: tener hijos adolescentes $(p=0,014)$ y realizar actividad académica $(p=0,005)$, presentó un factor de alto riesgo para el estrés. Conclusiones: Se observó que aproximadamente la mitad de los entrevistados presentaba estrés $(44,2 \%)$ y la mayoría se encontraba en fase de resistencia con un síntoma de factor psicológico $(60,9 \%)$. Presentar las variables: tener hijos adolescentes $(p=0,014)$ y realizar actividad académica $(p=0,005)$, presentó un factor de alto riesgo para el estrés.

Palabras clave: Enfermeras practicantes, Estrés laboral, Centros quirúrgicos.

\section{INTRODUÇÃO}

Aponta-se que o estresse está cada vez mais presente em inúmeras profissões e os profissionais da saúde, especificamente a enfermagem, incluem-se em uma das classes de trabalho mais prejudicadas. Por estarem diretamente envolvidos como cuidado, a manutenção da qualidade de vida e a promoção de bemestar dos indivíduos, muitas vezes abandonam seu próprio autocuidado, fato esse que pode afetar sua saúde emocional e física. Esse dado deve-se ao fato de que no ambiente laboral, no qual este tipo de profissional atua, existem vários estressores que influenciam significativamente e em destaque evidencia-se a sobrecarga de trabalho, a realização de tarefas simultâneas e a falta de materiais em grande parte das instituições (PUERTO JC, et al., 2017).

Alguns autores defendem a ideia de que os estressores não geram o estresse sozinho. Tais teóricos entendem que depende da interpretação que o indivíduo faz a respeito do estressor e que a partir dessa, o mesmo torna-se fator condicionante capaz de gerar o estresse. Dessa forma, a maneira como o profissional lida, interpreta e compreende os eventos ocorridos no ambiente de trabalho é o principal fator desencadeante ou não do estresse (NEUFELD CB e RANGÉ BP, 2017).

O ambiente hospitalar é considerado por diversos autores como estressante para os profissionais da saúde. De fato, alguns estressores são vivenciados nesse local como, por exemplo, sofrimento, angústia, condições de trabalho, demandas de assistência e alta responsabilidade. Sabe-se que o hospital é dividido em unidades, que cada uma possui as suas particularidades e considera-se que essa questão pode influenciar na percepção do profissional a respeito do estresse e dos estressores (SILVA JM e MALAGRIS LEN, 2019; GUIDO LA, et al., 2011).

Alguns setores hospitalares estão mais propensos ao um maior nível de estresse devido a sua complexidade: Unidade de Terapia Intensiva (UTI), Centro Cirúrgico (CC) e Emergências. Esses setores exigem cuidados específicos com maior segurança nos procedimentos e atenção constante nos parâmetros de vida do paciente. Todas essas situações manifestam momentos de maior tensão na equipe envolvida que deve manter consequentemente a segurança do cliente e a qualidade do serviço prestado (SOUZA JD, et al., 2017).

Os profissionais que atuam em unidades caracterizadas por demandas de alta complexidade convivem diariamente com a dor, o sofrimento e, muitas vezes, maior risco de morte dos indivíduos sob seus cuidados. Esses colaboradores têm um alto risco de desenvolver estresse psicológico e físico, podendo impactar na sua qualidade de vida e em seu desempenho profissional (ACIOLI NF, et al., 2013). 
Trabalhadores de centros cirúrgicos possuem grandes responsabilidades pela demanda de atividades específicas do ambiente associada, por vezes, aos recursos humanos insuficientes, falta de equipamentos ou materiais necessários à assistência prestada ao paciente cirúrgico. Muitas vezes profissionais de enfermagem que atuam em CC desenvolvem desgastes físicos, alterações emocionais e afastamento de relações sociais. Dessa forma, os serviços de saúde necessitam efetuar medidas para reduzir o estresse nesses ambientes de trabalho e na infraestrutura da unidade, incentivar programas de atenção à saúde do trabalhador, bem como a organização racional e valorização do trabalho (BRANDÃO DEC e GALVÃO CM, 2013).

A International Labour Organizatio (ILO) realizou, em 2016, uma pesquisa com especialistas de 54 países, obtendo como resultado a indicação em $90 \%$ dos participantes de que o estresse é um problema global. Dessa forma, os riscos psicossociais do estresse aumentaram o interesse de pesquisadores das áreas da saúde para realizarem estudos sobre o tema (ILO, 2016).

A definição de estresse pode ser compreendida como um estado de desequilíbrio do funcionamento diante de situações desafiadoras. Esses eventos levam o organismo a utilizar seus recursos psicobiológicos a fim de reconstituir o equilíbrio. No entanto, elevados níveis de estresse podem gerar enfermidades físicas como doenças cardiovasculares e transtornos mentais, tais como a síndrome Burnout, ansiedade e depressão (NEUFELD CB e RANGÉ BP, 2017; ILO, 2016).

Sabe-se que os profissionais da enfermagem possuem índice de estresse mais elevados quando comparados a outros profissionais (PUERTO JC, et al., 2017; ALMEIDA AMO, et al., 2016; KIRHHOF RS, et al., 2016 e SORATTO MT, et al., 2016). As práticas dos trabalhadores de enfermagem que atuam nos centros cirúrgicos geram muita tensão, pois lidam diariamente com procedimentos estressantes relacionados à gravidade dos pacientes sob seus cuidados, complexidade dos procedimentos cirúrgicos, pressão exercida por outros profissionais da equipe de saúde, falta de materiais e estrutura física inadequada às necessidades laborais. Essas situações vivenciadas diariamente influenciam negativamente na vida desses colaboradores gerando estresse e desmotivação, afetando o clima organizacional e bom desempenho da equipe (PASSOS JB, et al., 2010).

Diante desse contexto faz-se necessário identificar sinais e sintomas de estresse em trabalhadores que atuam em centro cirúrgico. A partir da hipótese que profissionais de enfermagem que atuam em áreas cirúrgicas possuem estressores contínuos que corroboram para ocorrência de sintomas de estresse tem-se como objetivo identificar qual é o nível de estresse dos trabalhadores de um centro cirúrgico a partir da implementação do Inventário de Sintomas de Estresse para adultos de lipp (ISSL).

\section{MÉTODOS}

Trata-se de uma pesquisa de abordagem transversal, descritiva e quantitativa, realizada em um Centro Cirúrgico (CC) e Centro de Materiais Esterilizados (CME) de um hospital de grande porte de uma cidade do RS. A amostra foi composta por profissionais de enfermagem, incluindo técnicos de enfermagem e enfermeiros. Para o cálculo amostral utilizou-se como base o artigo intitulado Análise do Estresse Ocupacional em Profissionais da Saúde (ASSIS MR, et al., 2015). De acordo com os estudos estatísticos preliminares, foi considerado em uma população de 62 profissionais, margem de erro de $5 \%$ e grau de confiança de $95 \%$, para este estudo a amostra conteve um total de 52 participantes.

A coleta de dados ocorreu no período de janeiro a junho do ano de 2020 e utilizou-se o Inventário de Sintomas de Estresse (ISS) para adultos. Trata-se de um teste autoaplicável composto por quadros característicos do estresse que possibilitam conhecer informações sobre o estresse em adultos e a fase em que a pessoa se encontra. Os sintomas listados no inventário são típicos de cada fase, sendo o instrumento um importante método de avaliação e medidas do nível de estresse.

Adicionado ao inventário foi aplicado um questionário semiestruturado, também autoaplicável, contendo informações sobre o perfil sociodemográfico dos trabalhadores: sexo, idade, estado civil, número de filhos, 
município de residência, profissão, cargo em exercício, setor de trabalho, tempo de serviço como profissional da enfermagem, realização de atividades acadêmicas e prática de atividades físicas.

No estudo foram incluídos colaboradores de ambos os sexos que realizavam atividades no Centro Cirúrgico e CME há mais de seis meses. Foram excluídos colaboradores que estavam afastados de suas atividades por motivo de licenças, atestados ou férias durante o período da coleta de dados da pesquisa.

Os dados quantitativos foram apresentados como média e desvio padrão (média \pm DP) e os categóricos por frequências absolutas e relativas ( $\mathrm{n}[\%]$ ). Para avaliar a associação entre as variáveis categóricas, o teste Qui-quadrado em conjunto com a análise dos resíduos ajustados foi utilizado. Para comparar médias, o teste t-student foi aplicado.

Para controle de fatores confundidores, o modelo de Regressão de Poisson com variância robusta foi aplicado. O critério para a entrada da variável no modelo multivariado foi de que a mesma apresentasse um valor $p<0,20$ na análise bivariada. O nível de significância adotado foi de $5 \%$ e as análises foram realizadas no Statistical Packages for the Social Sciences (SPSS) Statistics versão 21.0 (SPSS Inc., Chicago, IL).

Para realização dessa pesquisa foi necessária à autorização institucional para realização da coleta de dados, através de termo de anuência. Após aprovação em Comitê de Ética em pesquisa sob o parecer no 3.942.759 e CAAE 29800719.4.0000.5665, os colaboradores foram convidados a participar do estudo, sendo esclarecidos a respeito dos objetivos, riscos e benefícios de sua participação, no qual assinaram um Termo de Consentimento Livre e Esclarecido (TCLE).

\section{RESULTADOS}

Participaram da pesquisa 52 profissionais de enfermagem, sendo 9 enfermeiros e 43 técnicos de enfermagem. Observou-se predominância do sexo feminino e idade média de 40,5 anos. A maioria dos participantes informou ser casado (a)ou coabitando com companheiro(a) $(71,2 \%)$, possuir filhos pequenos $(36,1 \%)$ ou em fase adolescência $(48,1 \%)$.

Dos profissionais da enfermagem houve uma prevalência de técnicos de enfermagem $(82,7 \%)$ que trabalhavam no centro cirúrgico $(71,2 \%)$. Quanto ao tempo de serviço, a maioria atuava na área da saúde entre 11 e 15 anos $(30,8 \%)$, possuía apenas um vínculo empregatício $(71,2 \%)$ com carga horária semanal de trabalho em 36 horas $(71,2 \%)$.

Não houve predominância de um específico turno de trabalho. Ainda, uma menor parte dos participantes relataram realizar atividade acadêmica $(19,2 \%)$ e metade dos entrevistados $(53,8 \%)$ relatou aderir a prática de atividade física diariamente (Tabela 1 ). 
Tabela 1 - Caracterização do perfil dos participantes que responderam ao inventário de sintomas de estresse (ISS).

\begin{tabular}{|c|c|}
\hline Variáveis & $\mathrm{n}=52$ \\
\hline Idade (anos) - média \pm DP & $40,5 \pm 11,4$ \\
\hline \multicolumn{2}{|l|}{ Sexo-n(\%) } \\
\hline Feminino & $42(80,8)$ \\
\hline Masculino & $10(19,2)$ \\
\hline \multicolumn{2}{|l|}{ Estado civil - $\mathrm{n}(\%)$} \\
\hline Com parceiro & $37(71,2)$ \\
\hline Sem parceiro & $15(28,8)$ \\
\hline \multicolumn{2}{|l|}{ Número de filhos - n(\%) } \\
\hline Zero & $17(32,7)$ \\
\hline Um & $17(32,7)$ \\
\hline Dois & $13(25,0)$ \\
\hline Três ou mais & $5(9,6)$ \\
\hline Tem filhos pequenos $-\mathrm{n}(\%)$ & $19(36,5)$ \\
\hline Tem filhos adolescentes $-n(\%)$ & $25(48,1)$ \\
\hline Reside em outro município - n(\%) & $24(46,2)$ \\
\hline \multicolumn{2}{|l|}{ Profissão - n(\%) } \\
\hline Enfermeiro & $9(17,3)$ \\
\hline Técnico de enfermagem & $43(82,7)$ \\
\hline \multicolumn{2}{|l|}{ Setor - n(\%) } \\
\hline Centro cirúrgico & $37(71,2)$ \\
\hline CME & $15(28,8)$ \\
\hline \multicolumn{2}{|l|}{ Tempo de profissão - n(\%) } \\
\hline$<5$ anos & $12(23,1)$ \\
\hline 6 a 10 anos & $7(13,5)$ \\
\hline 11 a 15 anos & $16(30,8)$ \\
\hline 16 a 20 anos & $7(13,5)$ \\
\hline$>20$ anos & $10(19,2)$ \\
\hline Possui mais de um vínculo empregatício - n(\%) & $15(28,8)$ \\
\hline \multicolumn{2}{|l|}{ Número de vínculos empregatícios - n(\%) } \\
\hline Um & $37(71,2)$ \\
\hline Dois & $13(25,0)$ \\
\hline Três ou mais & $2(3,8)$ \\
\hline \multicolumn{2}{|l|}{ Carga horária semanal - n(\%) } \\
\hline $36 \mathrm{~h}$ & $37(71,2)$ \\
\hline $72 \mathrm{~h}$ & $13(25,0)$ \\
\hline Acima de $72 \mathrm{~h}$ & $2(3,8)$ \\
\hline \multicolumn{2}{|l|}{ Turno - n(\%) } \\
\hline Manhã & $11(21,2)$ \\
\hline Tarde & $13(25,0)$ \\
\hline Noite & $13(25,0)$ \\
\hline Mais de 1 turno & $15(28,8)$ \\
\hline Realiza atividade acadêmica $-\mathrm{n}(\%)$ & $10(19,2)$ \\
\hline Realiza atividade física - n(\%) & $28(53,8)$ \\
\hline
\end{tabular}

Na pesquisa verificou-se que a maioria dos participantes não apresentou sintomas de estresse $(55,8 \%)$. Contudo, daqueles profissionais que apresentavam os sintomas compatíveis $(44,2 \%)$ a maior parte encontrava-se em fase de resistência $(60,9 \%)$ e predominância do sintoma fator psicológico $(60,9 \%)$, conforme resultados apresentados (Tabela 2). 
Tabela 2 - Frequência sobre sintomas e níveis de estresse entre os participantes que responderam ao inventário de sintomas de estresse (ISS).

\begin{tabular}{lc}
\hline Variáveis & $\mathbf{n = 5 2}$ \\
\hline Estresse - $\mathbf{n}(\%)$ & $23(44,2)$ \\
\hline Sim & $29(55,8)$ \\
Não & $\mathbf{n = 2 3}$ \\
\hline- & \\
\hline Fase do estresse - $\mathbf{n}(\%)$ & $2(8,7)$ \\
\hline Alerta & $14(60,9)$ \\
Resistência & $6(26,1)$ \\
Quase exaustão & $1(4,3)$ \\
Exaustão & $43,2 \pm 21,2$ \\
\hline Físico (\%) - média $\mathbf{D}$ DP & $56,5 \pm 30,6$ \\
\hline Psicológico (\%) - média \pm DP & $6(26,1)$ \\
\hline Sintomas predominantes - $\mathbf{n ( \% )}$ & $14(60,9)$ \\
\hline Físicos & $3(13,0)$ \\
\hline Psicológicos &
\end{tabular}

Fonte: Dapper ASS, et al., 2020.

Ao comparar os trabalhadores com e sem estresse percebeu-se que os com sintomatologia presente apresentaram idade média de 36,8 (DP $\pm 7,8$ ), prevalência do sexo feminino (95,7\%), com companheiro $(78,3 \%)$ e sem filhos (43,5\%). Já os sem sintomas de estresse apresentaram idade média de 43,4 (DP $\pm 13,1)$, prevalência do sexo feminino $(69,0 \%)$, com parceiro $(65,5 \%)$ e com dois filhos $(37,9 \%)$.

Observou-se associação estatisticamente significativa entre o estresse e a variável número de filhos. Na avaliação dos trabalhadores com estresse e com filhos a maioria eram crianças pequenas $(43,5 \%)$ enquanto, nos trabalhadores sem estresse e com filhos, os maiores percentuais relacionavam-se à fase de adolescência $(62,1 \%)$.

O fato de ter dois filhos parece proteger os trabalhadores com o estresse apresentando uma variável de $(37,9 \%)$. Outro fator que apresenta maior nível de estresse é o fato de realizar atividade acadêmica $(30,4 \%)$, conforme dados apresentados abaixo (Tabela 3). 
Tabela 3 - Avaliação do perfil dos participantes com e sem sintomas de estresse que responderam ao inventário de sintomas de estresse (ISS).

\begin{tabular}{|c|c|c|c|}
\hline Variáveis & $\begin{array}{c}\text { Com estresse } \\
(n=23)\end{array}$ & $\begin{array}{c}\text { Sem estresse } \\
(n=29)\end{array}$ & $\mathbf{P}$ \\
\hline Idade (anos) - média \pm DP & $36,8 \pm 7,8$ & $43,4 \pm 13,1$ & 0,038 \\
\hline Sexo-n(\%) & & & 0,030 \\
\hline Feminino & $22(95,7)$ & $20(69,0)$ & \\
\hline Masculino & $1(4,3)$ & $9(31,0)$ & \\
\hline Estado civil - $n(\%)$ & & & 0,484 \\
\hline Com parceiro & $18(78,3)$ & $19(65,5)$ & \\
\hline Sem parceiro & $5(21,7)$ & $10(34,5)$ & \\
\hline Número de filhos - $\mathrm{n}(\%)$ & & & 0,037 \\
\hline Zero & $10(43,5)$ & $7(24,1)$ & \\
\hline Um & $7(30,4)$ & $10(34,5)$ & \\
\hline Dois & $2(8,7)$ & $11(37,9)^{*}$ & \\
\hline Três ou mais & $4(17,4)$ & $1(3,4)$ & \\
\hline Tem filhos pequenos $-\mathrm{n}(\%)$ & $10(43,5)$ & $9(31,0)$ & 0,525 \\
\hline Tem filhos adolescentes $-n(\%)$ & $7(30,4)$ & $18(62,1)$ & 0,047 \\
\hline Reside em outro município - $\mathrm{n}(\%)$ & $13(56,5)$ & $11(37,9)$ & 0,291 \\
\hline Profissão - n(\%) & & & 1,000 \\
\hline $\begin{array}{l}\text { Enfermeiro } \\
\text { Técnico de enfermagem }\end{array}$ & $4(17,4)$ & $5(17,2)$ & \\
\hline $\begin{array}{l}\text { Iecnico de entermagem } \\
\text { Setor }-\mathbf{n}(\%)\end{array}$ & $19(82,6)$ & $24(82,8)$ & 1,000 \\
\hline Centro cirúrgico & $16(69,6)$ & $21(72,4)$ & \\
\hline CME & $7(30,4)$ & $8(27,6)$ & \\
\hline Tempo de profissão - n(\%) & & & 0,248 \\
\hline$<5$ anos & $6(26,1)$ & $6(20,7)$ & \\
\hline 6 a 10 anos & $2(8,7)$ & $5(17,2)$ & \\
\hline 11 a 15 anos & $10(43,5)$ & $6(20,7)$ & \\
\hline 16 a 20 anos & $3(13,0)$ & $4(13,8)$ & \\
\hline$>20$ anos & $2(8,7)$ & $8(27,6)$ & \\
\hline Possui mais de um vínculo empregatício - n(\%) & $7(30,4)$ & $8(27,6)$ & 1,000 \\
\hline Número de vínculos empregatícios - n(\%) & & & 0,970 \\
\hline Nenhum & $16(69,6)$ & $21(72,4)$ & \\
\hline Dois & $6(26,1)$ & $7(24,1)$ & \\
\hline Três ou mais & $1(4,3)$ & $1(3,4)$ & \\
\hline Carga horária semanal - n(\%) & & & 0,258 \\
\hline $36 \mathrm{~h}$ & $16(69,6)$ & $21(72,4)$ & \\
\hline $72 \mathrm{~h}$ & $5(21,7)$ & $8(27,6)$ & \\
\hline Acima de $72 \mathrm{~h}$ & $2(8,7)$ & $0(0,0)$ & \\
\hline Turno - n(\%) & & & 0,950 \\
\hline Manhã & $4(17,4)$ & $7(24,1)$ & \\
\hline Tarde & $6(26,1)$ & $7(24,1)$ & \\
\hline Noite & $6(26,1)$ & $7(24,1)$ & \\
\hline Mais de 1 turno & $7(30,4)$ & $8(27,6)$ & \\
\hline Realiza atividade acadêmica $-\mathrm{n}(\%)$ & $7(30,4)$ & $3(10,3)$ & 0,087 \\
\hline Realiza atividade física - $n(\%)$ & $9(39,1)$ & $19(65,5)$ & 0,106 \\
\hline
\end{tabular}

Legenda: * associação estatisticamente significativa pelo teste dos resíduos ajustados a $5 \%$ de significância. Fonte: Dapper ASS, et al., 2020.

Para controle de fatores confundidores o modelo de Regressão de Poisson multivariado foi aplicado, permanecendo, estatisticamente significativo, as variáveis: ter filhos adolescentes $(p=0,014)$ e realiza atividade acadêmica $(p=0,005)$, como fator de risco elevado ao estresse (Tabela 4$)$. 
Tabela 4 - Análise de Regressão de Poisson multivariada para avaliar fatores independentemente associados ao estresse.

\begin{tabular}{lccc}
\hline Variáveis & Razão de Prevalências (RP) & IC 95\% & P \\
\hline Idade (anos) & 0,99 & $0,96-1,02$ & 0,647 \\
\hline Sexo & & & \\
\hline Feminino & 6,56 & $0,93-46,2$ & 0,059 \\
$\quad$ Masculino & 1,00 & - & - \\
\hline Número de filhos & & & - \\
$\quad$ Zero & 1,00 & - & 0,689 \\
Um & 0,87 & $0,45-1,69$ & 0,436 \\
Dois & 0,55 & $0,12-2,48$ & 0,210 \\
Três ou mais & 2,80 & $0,56-13,9$ & $\mathbf{0 , 0 1 4}$ \\
Tem filhos adolescentes & $\mathbf{0 , 4 7}$ & $\mathbf{0 , 2 6 - \mathbf { 0 , 8 6 }}$ \\
\hline Realiza atividade acadêmica & $\mathbf{1 , 9 5}$ & $\mathbf{1 , 2 3 - 3 , 1 0}$ & $\mathbf{0 , 0 0 5}$ \\
\hline Realiza atividade física & 0,68 & $0,39-1,19$ & 0,177 \\
\hline
\end{tabular}

Fonte: Dapper ASS, et al., 2020.

Por fim, a tabela cima demonstra-nos que os profissionais com filhos adolescentes possuem uma redução média de $53 \%$ na probabilidade de estresse. Também, os que realizam atividades acadêmicas, apresentaram uma probabilidade cerca 2 vezes maior de estresse do que os que não realizam essa atividade.

\section{DISCUSSÃO}

No presente estudo foi observado predominância por pessoas do sexo feminino, fato relacionado à profissão enfermagem ser majoritariamente conduzida por mulheres. Esta afirmação foi observada através de dados apresentados por um estudo, realizado em 2015, pelo Conselho Federal de Enfermagem (COFEN) e que caracterizou o perfil dos trabalhadores de enfermagem no Brasil concluindo que $80,7 \%$ dos profissionais são do gênero feminino (COFEN, 2015).

Pesquisa recente destacou que a prevalência do sexo feminino na profissão enfermagem implica em considerável relevância no que se refere aos fatores estressores e ocorre porque grande parte das mulheres necessitam conciliar as atividades domésticas, vida conjugal e cuidado com os filhos. Também, por estarem em idade produtiva, acabam somando a necessidade de melhorar sua qualificação profissional, condição de vida e assim buscam, muitas vezes, retomar os estudos para melhores oportunidades de trabalho (BARBOZA MCN, et al., 2016).

A presente pesquisa demonstrou dados relacionados às pessoas com níveis de estresse vinculados ao fato de realizarem atividades acadêmicas concomitantes com o trabalho destacando que, aqueles que estudam apresentam probabilidade duas vezes maior de desenvolverem estresse ao serem comparados com os que não exercem atividades de formação ou especialização. Confirmando estes dados, uma pesquisa publicada por Cestari VRF, et al. (2017) apresentou achados semelhantes relacionados aos estudos de formação exercidos simultaneamente com atividades profissionais. Os achados apontaram que os estudantes que exercem atividades remuneradas apresentaram maiores chances de adquirir estresse quando comparados aos que não necessitava trabalhar. Destaca-se que, de fato, a soma do trabalho com estudos requer maiores responsabilidades com as atividades diárias, com as tarefas universitárias, com o cuidar do lar, da família e dos filhos. Em Portugal, Santos LR e Veiga FR (2012), realizaram uma pesquisa com estudantes do ensino superior e observaram que15,0\% foram classificados com indicativo de depressão. A sintomatologia mais elevada ocorreu em estudantes do sexo feminino vinculados aos cursos da área da saúde e níveis socioeconômicos mais baixos, fato esse que influencia bastante no dia a dia.

Outro fator importante observado relaciona-se à situação conjugal e a presença de filhos, pois a maioria dos trabalhadores da presente pesquisa apresentava situação conjugal com companheiro (a) e com filhos pequenos ou em fase de adolescência. Estudos semelhantes indicam que estudantes solteiros e sem filhos conseguem ter uma melhor dedicação aos estudos (CESTARI VRF, et al., 2017 e COSTA CRB, et al., 2018). 
Além disso, pesquisa publicada no ano de 2012 concluiu que pessoas casadas ou que possuem vida conjugal estável associada ao fato de possuir filhos, apresentaram menor propensão ao desenvolvimento de estresse crônico (FRANÇA SPS, 2012). No entanto, na pesquisa de Cestari VRF, et al. (2017) os estudantes que declararam ter, pelo menos, um filho ou mais apresentavam 3,66 mais chances de desenvolverem estresse. Outro estudo publicado em 2015 apresentou dados divergente comparado ao estudo citado anteriormente, no qual foi observado o maior nível de estresse naqueles indivíduos que não possuíam filhos (CAMARGO VCV, et al, 2015).

O estudo de Santana LC, et al. (2019) analisou a relação da exposição ao estresse e a situação conjugal concluindo que, os maiores índices de estresse relacionavam-se às pessoas solteiras ou que possuem filhos, profissionais que exercem cargo de nível superior, aqueles com regime trabalhista estatutário e, também, os profissionais que realizam suas atividades no turno noturno.

A presente pesquisa conseguiu analisar a relação entre estresse e número de filhos. Nela, os profissionais que possuem apenas um filho apresentaram maior propensão ao estresse quando comparado com os profissionais que apresentam dois filhos ou mais. Este fato pode estar relacionado a seguinte situação: quando a pessoa tem dois filhos com idades próximas, um acaba sendo companheiro do outro, ou seja, brincam e interagem juntos. Porém, quando se tem apenas um filho esse papel é exercido pelos pais que muitas vezes, necessitam dividir a atenção lúdica com as demandas diárias do lar ou trabalho. A partir destes dados foi possível observar que o fator dois filhos ou mais parece proteger os trabalhadores contra o estresse, sendo merecedor demais pesquisas.

Observando os estudos já realizados, percebeu-se que há divergências em relação aos resultados que se referem ao número de filhos. Os estudos anteriores destacam que na análise da relação número de filhos e estresse, possuir ou não filhos pode diminuir ou aumentar o estresse. Contudo, estes estudos não especificaram se a quantidade de filhos é um fator relevante para aumento dos índices (FRANÇA SPS, 2012; CESTARI VRF, et al., 2017; COSTA CRB, et al., 2018 e SANTANA LC, et al., 2019).

Nesta pesquisa outra variável importante observada está relacionada a presença de filhos adolescentes, pois os achados demonstram importante relação com a redução da probabilidade de estresse. No entanto, para corroborar ou refutar esta análise destaca-se que não foram encontrados achados específicos que possam relacionar o número de filhos ou filhos em fase de adolescência como fator de proteção ao estresse. Neste sentido, ao pensarmos sobre os percentuais deduzimos que, ter um filho exige maior dedicação e atenção dos pais. Já, quando se tem dois filhos ocorre interação entre eles através de brincadeiras e outras formas de atenção, possivelmente exigindo menor resguardo ou dedicação integral dos pais. Acreditamos que um estudo qualitativo poderia auxiliar na resolução destas hipóteses.

Algumas características sociodemográficas e acadêmicas podem influenciar no nível do estresse, sendo elas: o sexo, a idade, estado civil e a existência de filhos (CACHOEIRA DVAC, et al., 2016). Na presente pesquisa a amostra foi constituída majoritariamente por profissionais técnicos de enfermagem. Resultados similares a este perfil profissional foi apresentado em outros estudos SANTANA LC, et al., 2019; PASSOS JB, et al., 2010; SOUZA VS, et al., 2018; RIBEIRO AC, e ROCHA R, 2018).

A presente pesquisa destacou que dentre os profissionais que apresentavam os sintomas compatíveis com estresse, a maioria encontrava-se em fase de resistência e apresentando predominância do sintoma fator psicológico. Outros estudos corroboram com os 29 dados encontrados demonstrado a fase de resistência e predominância do sintoma fator psicológico em ênfase (SANTANA LC, et al., 2019; PASSOS JB, et al., 2010; COSTA CRB, et al., 2018 e KESTENBERGI CCF, et al., 2017).

Destaca-se que é na fase de resistência que são utilizadas reservas de energia para haver um equilíbrio no organismo, ou seja, é nesta fase acontece uma ação reparadora do organismo na tentativa de restabelecer o equilíbrio interno. Portanto, trata-se de uma fase que pode ser de longa duração (SELYE H, 1965; LIPP MEN e GUEVARA AJH, 1994). Ainda, a prevalência dos sintomas psicológicos também foi um resultado bastante considerável e, de acordo com a literatura, algumas manifestações psicológicas se destacam, como por exemplo: a raiva, a baixa autoestima e a ansiedade (ALZAYYAT A e AI-GAMAL E, 2014). 
Esta pesquisa analisou especificamente profissionais que trabalham em setores específicos, ou seja, bloco cirúrgico e CME. Estudos destacam que os setores considerados específicos possuem maior propensão ao estresse, em especial aos profissionais que atuam em bloco cirúrgico (SANTANA LC, et al., 2019; PASSOS JB, et al., 2010). Porém, devemos considerar que os achados das outras pesquisas que corroboram com este estudo foram realizados em hospitais gerais, o que pode influenciar nos dados quando comparados a hospitais que atendem uma só especialidade, pois a presente pesquisa foi realizada especificamente em um o centro cirúrgico cardiológico.

Destaca-se como limitação que a presente pesquisa foi realizada em um centro cirúrgico de uma especialidade específica e permitiu que o pesquisador explorasse apenas uma única realidade. Tal fato permite a indagação a ser explorada: o serviço que exerce uma única especialidade pode influenciar em relação aos níveis de estresse? Diante destas discussões, seria interessante novos estudos realizados em setores específicos e com especialidades específicas, permitindo a observação de resultados semelhantes ou divergentes.

\section{CONCLUSÃO}

O estudo permitiu traçar o perfil dos trabalhadores que estão mais predispostos ao stress, demostrando que os trabalhadores do CC e CME apresentaram elevados índices de estresse em fase de resistência. A idade, o sexo, o número de filhos e ter filhos adolescentes demonstraram estar associados a ocorrência de stress. Entretanto, após implementação do modelo ajustado, permaneceram, estatisticamente significativas, as variáveis: ter filhos adolescentes e realizar atividade acadêmica como fator de risco para o estresse. Acredita-se que tal situação possa estar relacionada às práticas dos profissionais que geram muita tensão, demostrando haver a necessidade de estratégias que ofereçam apoio à realização de atividade profissional nesses setores.

\section{REFERÊNCIAS}

1. ACIOLI NA, et al. Qualidade de vida e nível de atividade física de profissionais de saúde de unidades de terapia intensiva. Ver Bras Ativ Fis e Saúd, 2013; 18(6): 711-71.

2. ALMEIDA AMO, et al. Estresse ocupacional em enfermeiros que atuam em cuidados ao paciente crítico. Revenferm UFPE online, 2016; 10(5): 1663-71.

3. ALZAYYAT A, AI-GAMAL E. A review of the literature regarding estresse among nursing students during their clinical education. Rev.IntNurs, 2014; 61(3): 406-15.

4. ASSIS MR, et al. Análise do Estresse Ocupacional em Profissionais da Saúde. Rio de Janeiro, 2015; 62-71.

5. BARBOZA MCN, et al. Estresse ocupacional em enfermeiros atuantes em setores fechados de um hospital de Pelotas/RS. RevEnferm UFSM, 2016; 3(3): 374-382.

6. BRANDÃO DEC, GALVÃO CM. Estresse da equipe de enfermagem que atua no período perioperatório: revisão integrativa. Rev Rene, 2013; 14(4): 836-44.

7. CACHOEIRA DVAC, et al. Relação do perfil sociodemográfico com o risco de adoecimento por transtornos mentais comum em alunos do curso de enfermagem. Rev Enferm UFPE, 2016; 10(12): 4501-8.

8. CAMARGO VCV, et al. Estresse, depressão e percepção de suporte familiar em estudantes de educação profissionalizante. Estudos de Psicologia. Campinas, 2015; 32(4): 595-604.

9. CESTARI VRF, et al. Estresse em estudantes de enfermagem: estudo sobre vulnerabilidades sociodemográficas e acadêmicas. Fortaleza, CE, Brasil. Acta Paul Enferm, 2017; 30(2): 190-6.

10. CONSELHO FEDERAL DE ENFERMAGEM (COFEN). O perfil da enfermagem no Brasil. Brasília: COFEN; 2015.

11. COSTA CRB, et al. Estresse entre estudantes de graduação em enfermagem: associação de características sociodemográficas e acadêmicas. Rev Saúde e Pesquisa, 2018;11(3): 475-482.

12. FRANÇA SPS, et al. Preditores da Síndrome de Burnout em enfermeiros de serviços de urgência pré-hospitalar. Acta Paul Enferm, 2012; 25(1): 68-73.

13. GUIDO LA. et al. Estresse, coping e estado de saúde entre enfermeiros hospitalares. Revista da Escola de Enfermagem da USP, 2011; 45(6): 1434- 1439.

14. INTERNATIONAL LABOUR ORGANIZATION (ILO). Workplace stress: A collective challenge. África: International Labour Office. 2016. Disponível em: https://www.ilo.org/wcmsp5/groups/public/---ed_protect/---protrav/--safework/documents/publication/wcms_466547.pdf. Acessado em: 11 de abril de 2020. 
15. KESTENBERG CCF, et al. Estresse em graduandos de enfermagem. Rev Enferm UERJ, 2017; $25:$ e26716.

16. KIRHHOF RS, et al. Nível de estresse entre enfermeiros de um hospital filantrópico de médio porte. Revista de Enfermagem da UFSM, 2016; 6(1): 29-39.

17. NEUFELD CB, RANGÉ BP. Terapia cognitivo comportamental em grupo: das evidências à prática. Porto Alegre, RS: Artmed, 2017; 326-338.

18. LIPP MEN, GUEVARA AJH. Validação empírica do Inventário de Sintomas de Estresse (ISS). Estudos de Psicologia (Campinas). 1994; 11(3): 43-9.

19. PASSOS JB, et al. Estresse no centro cirúrgico: uma realidade dos profissionais de enfermagem. Revista Pesq Saúde, 2010; 11(2): 35-38.

20. PUERTO JC, et al. Uma nova contribuição para a classificação dos fatores estressores que afetam os profissionais de enfermagem. Rev. Latino-Am. Enfermagem, 2017; 25: e2895.

21. RIBEIRO AC, et al. Fatores do estresse ocupacional na equipe de enfermagem: uma revisão integrativa. Rev Eletrônica do Univag - Connection line. 2018; 19: 98-105.

22. SANTANA LL, et al. Estresse no Cotidiano de Graduandos de Enfermagem de um Instituto Federal de Ensino, 2018; 8: e2738.

23. SANTANA LC, et et al. Occupational estresse in nursing professionals of a university hospital. Rev Bras Enferm, 2019; 73(2): e20180997.

24. SANTOS LR, et al. Sintomatologia depressiva e percepção do rendimento académico no estudante do ensino superior. Actas do 12ำ Colóquio de Psicologia - Educação, Aprendizagem e Desenvolvimento: olhares contemporâneos através da investigação e da prática. Lisboa: ISPA, 2012.

25. SELYE H. The Estresse Syndrome. AJN The American Journal of Nursing, EUA, 1965; 65(3): 97-99.

26. SILVA JM, MALAGRIS LEN. Percepção do estresse e estressores de enfermeiros de um hospital universitário. Estudos e Pesquisas em Psicologia, 2019; 19(1): 71-88.

27. SORATTO MT, et al. O Estresse da equipe de enfermagem no centro cirúrgico, 2016; 5(1): 179-192.

28. SOUZA JD. Estresse e em serviço de urgência e os desafios para enfermeiros brasileiros e portugueses. Revista de Enfermagem Referência, Coimbra, 2017; 12: 107-116.

29. SOUZA VS, et al. Qualidade de vida dos profissionais de enfermagem atuantes em setores críticos. Rev Cuid, 2018; $9(2): 2177-86$. 